\title{
Incarcerated Omentum With Tamponade Effect in the Uterine Perforation Scar After Dilation and Curettage: A Case Report
}

\author{
Myounghwan Kim a, b
}

\begin{abstract}
Perforation of the uterus is the most common immediate complication at the time of dilatation and curettage (D\&C). We experienced the case of a patient with intra-abdominal extrusion of fetal part and incarcerated omentum in the perforation site of uterine fundus after D\&C. It resulted in massive bleeding after the reduction of the incarcerated omentum which had a tamponade effect. A 26-yearold gravida 2, para 1 woman was referred to our hospital because of uterine perforation during D\&C. A laparoscopic exploration was performed. Bleeding started massively at the perforation scar after reduction of the incarcerated omentum which had a tamponade effect. Though bleeding from perforation site stopped after laparoscopic myometrial repair, vaginal bleeding persisted further. The placing of an intrauterine balloon tamponade stopped the vaginal bleeding. The patient recovered successfully and was discharged on the fifth postoperative day.
\end{abstract}

Keywords: Dilatation and curettage; Extrusion of fetal part; Incarcerated omentum; Tamponade effect; Uterine perforation

\section{Introduction}

Perforation of the uterus at the time of dilatation and curettage $(\mathrm{D} \& \mathrm{C})$ is the most common immediate complication. The risk of perforation is increased by factors that make access to the endometrial cavity difficult (for example, cervical stenosis) or alter the strength of the myometrial wall (for example, pregnancy, menopause). Most uterine perforations escape medical detection without hemorrhage or visceral in-

\footnotetext{
Manuscript accepted for publication February 18, 2014

${ }^{a}$ Department of Obstetrics and Gynecology, Sanggye Paik Hospital, Inje University, School of Medicine, Seoul, Korea

${ }^{\mathrm{b}}$ Corresponding author: Myounghwan Kim, Inje University, Sanggye

Paik Hospital, Sanggye 7 dong 761-1, Nowon-gu, Seoul 139-707,

Korea. Email: myankim@paik.ac.kr
}

doi: http://dx.doi.org/10.14740/jmc1713w jury [1]. Perforation at the fundus is most common and typically leads to minimal bleeding, though it occurs, whereas a lateral uterine perforation may lacerate uterine blood vessels, thus resulting in immediate and profuse hemorrhage and, possibly, a broad ligament hematoma. We experienced the case of a patient with incarcerated omentum in the perforation site of uterine fundus after $\mathrm{D} \& \mathrm{C}$, resulting in massive bleeding after the reduction of the incarcerated omentum which had a tamponade effect. It was treated with laparoscopic suture and intrauterine balloon tamponade.

\section{Case Report}

A 26-year-old gravida 2, para 1 woman was referred to our hospital because of uterine perforation during D\&C. The patient underwent a $\mathrm{D} \& \mathrm{C}$ for elective termination of pregnancy at week 11. The patient complained of lower abdominal pain and the physical examination revealed diffuse abdominal tenderness and rebound tenderness. Vital signs were measured with blood pressure $117 / 59 \mathrm{mmHg}$, pulse rate 73 beats per minute and body temperature $36.5^{\circ} \mathrm{C}$. The serum hemoglobin was $13.0 \mathrm{~g} / \mathrm{dL}$, hematocrit was $37 \%$ and the leukocyte level was $8,900 / \mu \mathrm{L}$. The transvaginal ultrasound scan demonstrated a thickened endometrium $(16 \mathrm{~mm})$. Both ovaries were normal; however, the pouch of Douglas was filled with

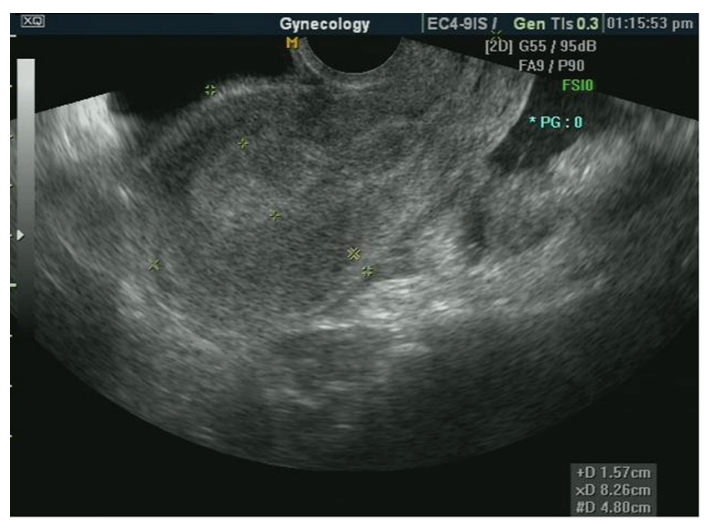

Figure 1. Ultrasound scan shows $1.9 \mathrm{~cm}$ of fluid collection in the pouch of Douglas. 


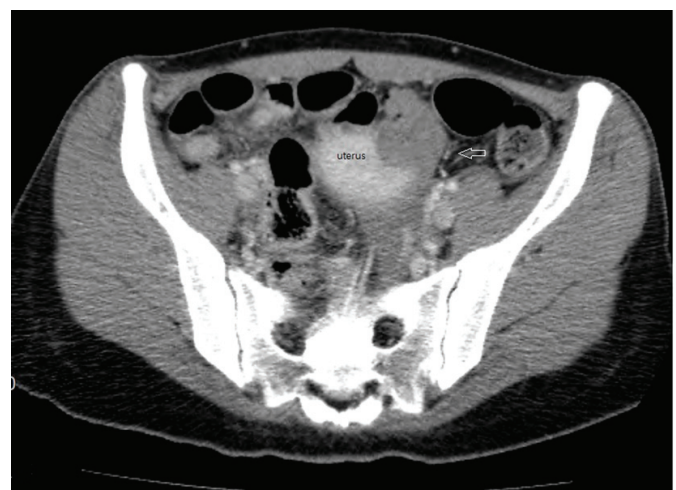

Figure 2. Abdomino-pelvic computed tomography shows hematoma around the perforation scar.

free fluid $(1.9 \mathrm{~cm})$ (Fig. 1). The abdomino-pelvic computed tomography (CT) showed no evidence of bowel injury except hematoma around perforation scar (Fig. 2). The vital signs remained stable during monitoring. The hemoglobin was $12.3 \mathrm{~g} / \mathrm{dL}$ and the fluid collection in the pouch of Douglas was $2.1 \mathrm{~cm}$ on ultrasound scan after $4 \mathrm{~h}$. After a sonoguided curettage of the remained conceptus in the uterine cavity, we decided to closely observe the patient. During the night, the body temperature was elevated and sustained be- tween 38.0 and $38.5^{\circ} \mathrm{C}$. On the following day, a laparoscopic exploration was performed. Upon entering the pelvic cavity, about $100 \mathrm{cc}$ of blood was noted in the pouch of Douglas. The omentum was incarcerated with a hematoma in the perforation scar of the uterine fundus, but a bowel injury was not found (Fig. 3A). To reduce the incarcerated omentum, it was pulled with a traumatic forcep. Bleeding started massively at the perforation scar after reduction of incarcerated omentum (Fig. 3B). A figure-of-eight suture at the perforation site stopped the bleeding. Fetal part was expelled into abdominal cavity (Fig. 3C) and a blood clot was evacuated with suction and forcep. Just as soon as we removed uterine manipulator, torrential vaginal bleeding commenced. Vaginal bleeding was stopped after one more deep figure-of-eight suture into the myometrium just at right side of previous stitch and placing an intrauterine balloon tamponade (Fig. 3D). The estimated amount of vaginal bleeding was about $800 \mathrm{cc}$. During the operation, four units of packed red blood cells were transfused. The patient recovered successfully and was discharged on the fifth postoperative day.

\section{Discussion}

$\mathrm{D} \& \mathrm{C}$ is a diagnostic and therapeutic surgical procedure used
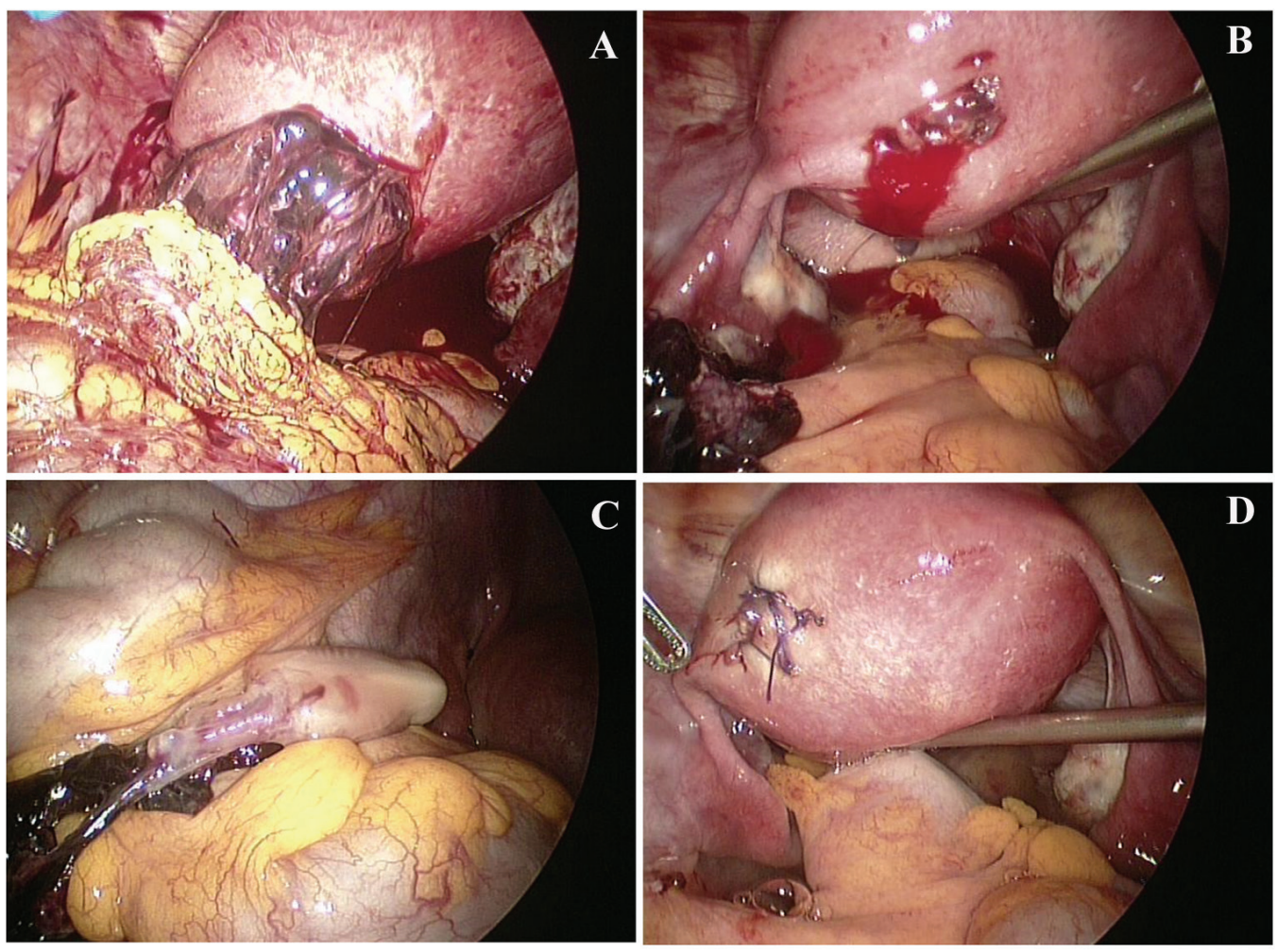

Figure 3. Laparoscopic view: about $100 \mathrm{cc}$ of blood in the pouch of Douglas and incarcerated omentum in the perforation scar of uterine fundus with hematoma (A), bleeding from perforation scar after removing incarcerated omentum (B), abdominal extrusion of fetal part (C) and after suturing the perforated scar (D). 
frequently throughout the world and is considered to be relatively safe with a low overall complication rate of $0.7 \%$ [2].

The rate of perforation varies with the indication for the procedure. Perforation is most common when attempting control of postpartum hemorrhage (5.1\%) and is less frequent during diagnostic curettage $(0.3 \%$ in the premenopausal patient and $2.6 \%$ in the postmenopausal patient) [3, 4]. Factors that make access to the endometrial cavity difficult or alter the strength of the myometrial wall predispose to uterine perforation, including [3] cervical stenosis; distortion or scarring of the endocervical canal (for example, due to cone biopsy); uterine malposition (for example, extreme retroversion or retroflexion); distortion of uterine anatomy (for example, due to fibroids, intrauterine adhesions); pregnancy; menopausal endometrial atrophy and myometrial thinning; menopausal vaginal atrophy and stenosis. In addition, a lack of surgical experience, extensive operative procedures (for example, resection of fibroids or intrauterine adhesions) and, for pregnancy-related procedures, underestimation of gestational age, have been associated with a higher risk of uterine perforation $[1,4,5]$.

A perforation should be suspected when a uterine sound, dilator or an operating instrument passes beyond the expected length of the uterus [6]. Ultrasound is often the initial diagnostic modality to be used for evaluation of uterine perforation. On sonography, the perforation may be implied by presence of indirect findings like visualization of bowel loops in myometrial or endometrial cavity or demonstration of extrauterine fetal parts in a pregnant female who has undergone a surgical abortion procedure recently. Sonography may sometimes be able to demonstrate the site of uterine rupture as a hypoechoic or anechoic transmural defect in myometrium extending to endometrium with presence of extrauterine fluid [2]. Usage of high resolution transvaginal probes can enhance the detection of perforation defects and mural hematomas [7]. CT has also been used for diagnostic evaluations of uterine perforations in few instances [8]. The site of perforation is seen as a hypoattenuating defect with disruption of myometrial continuity on the CT.

Relative avascular anterior or posterior midline surfaces are the most common sites of myometrial perforations in uterine surgeries [8]. Most uterine perforations escape a medical detection without hemorrhage or visceral injury [1]. Perforations are more likely to be troublesome if the perforation is laterally located, the defect is more than $1.2 \mathrm{~cm}$, they occur after first trimester, or they are associated with a bowel injury. In most of cases related with abortion, the perforation is recognized by the operator during the procedure. However, in many cases, perforations may remain clinically undiagnosed and the patients were discharged. Some of these patients present subsequently with serious complications [9].

A uterine perforation that is not complicated by bleeding or injury to surrounding structures may require observation only, while a more involved perforation is a surgical emergency. Abdominal exploration should be immediately performed if there are signs of severe uterine bleeding or a vascular or visceral injury is suspected.

Among patients who require abdominal exploration, laparoscopy is preferred if the patient is stable since the risks of perioperative morbidity are lower. However, it is difficult to evaluate the entire bowel at laparoscopy and an experienced operative laparoscopist is needed to accomplish both evaluation and repair using laparoscopic instruments.

In our present case, bleeding and imaging studies were not serious, but persistent fever, abdominal tenderness and rebound tenderness led us to perform a laparoscopic exploration. Uterine perforation was complicated with omental incarceration and intra-abdominal extrusion of the fetal part. Bleeding started massively at the perforation scar after reduction of incarcerated omentum, which had a tamponade effect. Though bleeding from perforation site stopped after laparoscopic myometrial repair, vaginal bleeding persisted. The placement of an intrauterine balloon tamponade stopped the vaginal bleeding. It seems that vaginal bleeding was due to vessel injury in endometrial cavity as well as due to bleeding in the perforation scar. In this situation, placing intrauterine balloon tamponade as well as figure-of-eight suturing deeply into myometrium is helpful to stop bleeding. An abdominal exploration is mandatory if an incarcerated omentum is suspected. However, Ozaki et al reported an asymptomatic incarcerated omentum caused by an evacuationrelated uterine perforation that was diagnosed 2 years after D\&C by accident during cesarean section [10]. The authors believe that similar cases of asymptomatic uterine perforation due to $\mathrm{D} \& \mathrm{C}$ may exist without definitive diagnosis.

In conclusion, laparoscopic exploration is the preferred method in the management of a patient with uterine perforation after D\&C. Suture of perforated scar should be performed deeply into the myometrium to stop the bleeding and also an intrauterine balloon tamponade is helpful.

\section{Conflict of Interest}

I did not have any financial support or relationships that may pose potential conflict of interest.

\section{References}

1. Amarin $\mathrm{ZO}$, Badria LF. A survey of uterine perforation following dilatation and curettage or evacuation of retained products of conception. Arch Gynecol Obstet. 2005;271(3):203-206.

2. Gakhal MS, Levy HM. Sonographic diagnosis of extruded fetal parts from uterine perforation in the retroperitoneal pelvis after termination of intrauterine pregnancy that were occult on magnetic resonance imaging. 
J Ultrasound Med. 2009;28(12):1723-1727.

3. Ben-Baruch G, Menczer J, Shalev J, Romem Y, Serr DM. Uterine perforation during curettage: perforation rates and postperforation management. Isr J Med Sci. 1980;16(12):821-824.

4. Grossman D, Blanchard K, Blumenthal P. Complications after second trimester surgical and medical abortion. Reprod Health Matters. 2008;16(31 Suppl):173-182.

5. Grimes DA, Schulz KF, Cates WJ, Jr. Prevention of uterine perforation during curettage abortion. JAMA. 1984;251(16):2108-2111.

6. Hefler L, Lemach A, Seebacher V, Polterauer S, Tempfer C, Reinthaller A. The intraoperative complication rate of nonobstetric dilation and curettage. Obstet Gynecol. 2009;113(6):1268-1271.

7. Sherer DM, Gorelick C, Gabbur N, Borowski D, Serur
E, Zinn HL, Kheyman M, et al. Transvaginal sonographic findings of a large intramural uterine hematoma associated with iatrogenic injury sustained at termination of pregnancy. Ultrasound Obstet Gynecol. 2007;30(1):110113.

8. Themistoklis SN, Chrysovalantis V, Stylianos A, Nikolaos KL, Efthymia A. CT Diagnosis of an Abortion-Related Retroperitoneal Space Abscess. J Clin Med Res. 2011;3(5):268-269.

9. Chauhan NS, Gupta A, Soni PK, Surya M, Mahajan SR. Iatrogenic uterine perforation with abdominal extrusion of fetal parts: a rare radiological diagnosis. J Radiol Case Rep. 2013;7(1):41-47.

10. Ozaki K, Suzuki S. Uterine perforation with omentum incarceration after dilatation and evacuation/curettage. Arch Gynecol Obstet. 2013;287(3):607-608. 